\title{
Determinação do ácido trans, trans-mucônico em urina: validação de um método analítico por cromatografia líquida de alta eficiência
}

\author{
Isarita Martins ${ }^{1 *}$, Maria Elisa Pereira Bastos de Siqueira² \\ 'Departamento de Análises Clínicas e Toxicológicas, Faculdade de Ciências Farmacêuticas, Universidade de São \\ Paulo, ${ }^{2}$ Departamento de Análises Clínicas e Toxicológicas, Escola de Farmácia e Odontologia de Alfenas, Centro \\ Universitário Federal
}

*Correspondência:

I. Martins

Departamento de Análises Clínicas e

Toxicológicas

FCF-USP

Av. Lineu Prestes, 580, bloco 13B 05389-970 - São Paulo - Brasil

E-mail: isarita@usp.br
A finalidade primordial da monitorização biológica é proteger a saúde dos trabalhadores, prevenindo a exposição excessiva às substâncias químicas. $O$ ácido trans,trans-mucônico (ttAM), um produto de biotransformação do benzeno, tem sido preconizado como um bioindicador sensível da exposição ao benzeno e este trabalho foi desenvolvido com o propósito de validar método de deteç̧ão do ttAM urinário, visando à sua utilização na biomonitorização de trabalhadores expostos a esse solvente. A técnica escolhida foi a cromatografia líquida de alta eficiência (CLAE) com coluna de fasereversa, Lichrosorb RP 18, e detector de ultravioleta. O método mostrou-se linear entre 0,2 a 5,0 mg/L $\left(r^{2}=0,9943\right)$. Os limites de detecção e de quantificação obtidos foram, respectivamente, 0,1 e $0,2 \mathrm{mg} / \mathrm{L}$. A porcentagem de recuperação absoluta média foi de 77,1\% e de inexatidão de 27,9\%. Os coeficientes de variação médios foram de $7,7 \%$ e $10,6 \%$ para a precisão intra-ensaio e interensaio, respectivamente. $O$ analito permaneceu estável na matriz por um período de 6 semanas para a concentração de $0,2 \mathrm{mg} / \mathrm{L}$ e de 15 semanas para a de 2,0 $\mathrm{mg} / \mathrm{L}$, se armazenada em freezer $\left(-20^{\circ} \mathrm{C}\right)$, $e$ por até dez dias sob refrigeração $\left(4^{\circ} \mathrm{C}\right)$ para os adicionados de 0,2 ; 2,0 e 5,0 mg/L. Com estes resultados, a validação foi considerada satisfatória e o método mostrou-se adequado, quando aplicado em amostras de trabalhadores que manipulam benzeno, coletadas no final da jornada de trabalho, em que se detectou um valor médio de 0,8 $\mathrm{mg} \mathrm{ttAM} / \mathrm{g}$ creatinina (mediana=0,6 $\mathrm{mg} / \mathrm{g}$ creatinina).

\section{INTRODUÇÃO}

A finalidade primordial da monitorização biológica é proteger a saúde dos trabalhadores, prevenindo a exposição excessiva às substâncias químicas. No entanto, na biomonitorização dos indivíduos expostos ao benzeno muitas são as dificuldades quanto ao uso de bioindicador adequado. Atualmente, os dois indicadores biológicos urinários que melhor avaliam a exposição ocupacional a baixos níveis de benzeno são o ácido trans,trans-mucônico (ttAM) e o ácido fenilmercaptúrico (Ong, Lee, 1994; Ghittori et al., 1995; Boogard, Van Sittert, 1995, 1996).

O ácido fenilmercaptúrico, apesar de ser específico e sensível, requer técnica de cromatografia gasosa acoplada 
à espectrometria de massas (Ong, Lee, 1994; Popp et al., 1994), o que ainda tem dificultado sua aplicação em análises rotineiras de biomonitorização no Brasil.

Assim, o ttAM em urina parece o mais promissor, e apesar de não ser específico (Medeiros et al., 1993; Ong et al., 1995; Weaver et al., 1996), apresenta boa correlação com o benzeno ambiental, mesmo em baixas concentrações (Buratti et al., 1996; Ong et al., 1996; Priante et al., 1996; Yu, Weisel, 1996; Kivisto et al., 1997).

Na determinação de ttAM, os métodos mais empregados são os que utilizam a cromatografia líquida de alta eficiência (CLAE). A detecção ocorre na faixa do ultravioleta ou pelo uso do espectrômetro de massas, e a separação, pelo uso de colunas cromatográficas de fase reversa, levando em conta a polaridade da substância a ser analisada, fato de importância na escolha dos componentes da fase móvel (Inoue et al., 1989; Johnston et al., 1991; Brondeau et al., 1992; Ducos et al., 1992; Ghittori et al., 1995; Boogard, Van Sittert, 1996).

Ultimamente, na tentativa de aperfeiçoar a análise, alguns autores optaram pela cromatografia gasosa associada à espectrometria de massas (CG-EM), que, apesar de bastante sensível e específica, torna a análise mais complexa e cara (Bechtold, Henderson, 1993; Ruppert et al., 1995).

Dentre as técnicas de separação e extração, as mais utilizadas são as que usam resinas de troca iônica (Brondeau et al., 1992; Maestri et al., 1995; Ghittori et al., 1996). Este processo melhorou o rendimento da recuperação em relação à extração líquido-líquido (Inoue et al., 1989; Bechtold et al., 1991).

Johnston et al. (1991) preconizam que o $\mathrm{pH}$, a força iônica e o tipo de tampão requeridos para a extração com tais resinas são muito importantes e influenciam na mobilidade do benzeno e seus metabólitos.

Em 1994, Bartczak et al. compararam os resultados encontrados na análise por cromatografia líquida e gasosa e concluíram que ambas apresentam boa correlação com valores de ttAM acima de $0,1 \mathrm{mg} / \mathrm{L}$ e que a cromatografia líquida só é limitada para a análise de concentrações inferiores a $0,04 \mathrm{mg} / \mathrm{L}$, devido à presença de picos interferentes nos cromatogramas.

A validação de um método analítico em cromatografia líquida de alta eficiência (CLAE) para determinação de ttAM em urina, bem como a avaliação de sua aplicação em amostras de trabalhadores que manipulam o benzeno, foram os objetivos deste trabalho.

\section{MATERIAL E MÉTODO}

\section{Reagentes e padrões}

-padrão de ácido trans,trans-mucônico 98\%-Sigma-
Aldrich $^{\circledR}$, lote n. ${ }^{\circ}$ 08809MR; padrão de ácido 4hidroxi-3-metoxibenzóico (ácido vanilico) 97\% Sigma-Aldrich ${ }^{\circledR}$, lote n. ${ }^{\circ}$ 04302MQ; resina Dowex 1x2-100 ion-exchange-chloride, strongly basic anion, 50-100 mesh- Sigma-Aldrich ${ }^{\circledR}$; acetato de sódio anidro p.a.; cloreto de sódio p.a.; ácido orto-fosfórico 85\% p.a.; tris(hidroximetil)aminometano- Sigma ${ }^{\circledR}$; metanol Omnisolv, grau de pureza cromatográfica-EM Science ${ }^{\circledR}$, ácido acético glacial $100 \%$ p.a.; ácido clorídrico $37 \%$ p.a. Para o preparo de soluções utilizou-se água de grau reagente (resistividade $>10$ megaohm), Millipore ${ }^{\circledR}$ (MilliQ).

- soluções padrão de ttAM: 1 g/L em água MilliQ (estoque). Esta solução deve ser preparada a cada mês e conservada a $-20^{\circ} \mathrm{C}$. As soluções de uso - de concentrações entre 0,2 e 5,0 mg/L, em urina e em água - foram preparadas a partir de uma solução a $20 \mathrm{mg} / \mathrm{L}$ em $\mathrm{HCl} 60 \mathrm{mmol} / \mathrm{L}$ (preparada semanalmente);

- solução padrão de ácido vanílico: 1 g/L em água MilliQ (estoque) e a $10 \mathrm{mg} / \mathrm{L}$ em tampão Tris a 0,5 mol/L (uso, preparada no dia da análise);

\section{Instrumentação}

- colunas para extração em fase sólida: colunas de vidro de $15 \mathrm{~cm}$ de altura, $1 \mathrm{~cm}$ de diâmetro interno e torneira de teflon. A coluna foi preparada com $300 \mathrm{mg}$ do adsorvente, Dowex $1{ }^{\circledR}$, suspensa em água Milli Q para a transferência, tendo-se o cuidado de compactar bem a resina, de modo a não deixar espaços sem preenchimento (o que pode ser conseguido pelo uso de vibrador e com vácuo);

- urodensímetro Urinometer $^{\circledR}$;

- kit para determinação de creatinina Labtest ${ }^{\circledR}$ - lote $n^{0}$ 80564;

- cromatógrafo líquido Hewlett Packard ${ }^{\circledR}$, modelo 1100, equipado com detector de UV, comprimento de onda= $259 \mathrm{~nm}$, acoplado a computador modelo Vectra XM, série 4-5/150, com ChemStation para integração e processamento dos cromatogramas, munido de coluna Chrompack $^{\circledR}$ - tipo Lichrosorb 5 RP18 (150x3 mm) e guarda-coluna Chrompack ${ }^{\circledR}$ R2 (10x2 mm), mantida a temperatura de $27^{\circ} \mathrm{C}$.

A fase móvel foi constituída de ácido acético 1\%/ metanol (9:1), filtrada sob pressão, através de filtro Millipore 0,45 mm, eluindo através da coluna, em um fluxo constante de $1 \mathrm{~mL} / \mathrm{min}$.

As técnicas de extração e identificação do ttAM em urina foram realizadas com base nos métodos propostos por Lee et al. (1993) e Ducos et al. (1990), respectivamente. O método utilizado pode ser descrito nas seguintes etapas: 
- em tubo de $15 \mathrm{~mL}$ misturar $1 \mathrm{~mL}$ de urina e $2 \mathrm{~mL}$ de tampão Tris $0,5 \mathrm{~mol} / \mathrm{L}$ ( $\mathrm{pH} 10)$, contendo ácido vanílico $10 \mathrm{mg} / \mathrm{L}$;

- transferir a mistura acima para coluna de vidro contendo a resina Dowex, pré-condicionada com $2 \mathrm{~mL}$ de água Milli Q, 2 vezes;

- lavar a coluna com $1 \mathrm{~mL}$ ácido fosfórico 0,2 mmol/L; $1 \mathrm{~mL}$ de tampão acetato de sódio 0,1 mol/L (pH 7) e $1 \mathrm{~mL}$ de água Milli Q;

- descartar todos os efluentes;

- em seguida, eluir o ttAM com 2 mL de uma mistura de cloreto de sódio 1,5 mol/L/metanol, na proporção 1:1 num fluxo de cerca de $1,0 \mathrm{~mL} / \mathrm{min}$, sem uso de vácuo;

- injetar $20 \mathrm{~mL}$ desse eluato no cromatográfo a líquido, segundo condições especificadas no item instrumentação.

A validação do método (Chasin et al., 1994; Chasin et al., 1998) foi realizada de acordo com os parâmetros:

- Linearidade: Para verificar a faixa de linearidade do método, foi adicionada às amostras de pool de urina, solução-padrão de ácido ttAM, de modo a obter as seguintes concentrações: 0,2; 0,5; 1,0; 2,0; 4,0; 5,0 mg/L. Estas amostras foram, assim como um branco de urina, analisadas em sextuplicata.

- Limite de quantificação (LQ): Foi considerado limite de quantificação, a menor concentração que se pode medir com precisão adequada, ou seja, com coeficiente de variação menor que $10 \%$.

- Limite de detecção (LD): Foi considerado limite de detecção, a concentração mínima que se diferenciou do zero, com determinada confiabilidade $(99,7 \%$ de confiança).

- Recuperação: Para avaliar a recuperação do método, foram utilizadas três concentrações: 0,2; 2,0 e 5,0 mg/L (sextuplicata/concentração). Por comparação da resposta quando a adição foi feita antes da extração versus a resposta quando a adição foi realizada após este procedimento, obteve-se a porcentagem de analito recuperado.

- Precisão: amostras de urina enriquecidas com 0,2; 2,0 e 5,0 mg/L de ttAM foram analisadas em 10 replicatas/ concentração, para o estabelecimento da precisão intra-ensaio e em triplicata, dia-após-dia, durante dez dias, para o estudo da precisão interensaio.

- Estabilidade do analito na matriz: A estabilidade foi avaliada em 45 amostras, enriquecidas com 0,2 e 2,0 mg/L de ttAM, conservadas a $-20^{\circ} \mathrm{C}$, sendo cada três analisadas semanalmente, por um período de 15 semanas. Urinas enriquecidas com 0,2; 2,0 e 5,0 mg/L de ttAM foram analisadas em 10 replicatas/concentração, durante 10 dias, para o estabelecimento da estabilidade das amostras em geladeira $\left(4^{\circ} \mathrm{C}\right)$.
- Exatidão: Para o estudo da exatidão, amostras de urina foram enriquecidas com ttAM, para se obter concentrações de 0,2; 2,0 e 5,0 mg/L (sextuplicata/ concentração). A inexatidão foi calculada pela tendenciosidade (bias).

- Efeito da matriz: Para avaliar o efeito da matriz biológica, ou seja, se mostrava a presença de outros constituintes que resultassem em picos próximos ou iguais ao do analito nas condições otimizadas, amostras de urina e de água foram enriquecidas, em duplicata, com as concentrações 0,$2 ; 0,5 ; 1,0 ; 2,0$ e 5,0 mg/L.

As amostras de urina destinadas à determinação de ttAM foram obtidas de trabalhadores que manipulam benzeno de uma refinaria, onde o petróleo passa por uma série de operações de beneficiamento até a obtenção de mais de 80 produtos de ampla utilização na indústria, como gás natural, gasolina, óleo diesel, solventes de borracha, asfalto, lubrificantes e o próprio benzeno. O trabalhador se expõe a este solvente na sua obtenção, na unidade de reforma catalítica, no manuseio, no transporte e no laboratório de controle de qualidade. O trabalho se faz segundo sistema de rodízio, ou seja, o trabalhador permanece na área onde existe benzeno apenas $1 \mathrm{dia} /$ semana, em turno de 6 h. Nesta área, trabalha usando equipamento completo de proteção individual. O projeto foi desenvolvido após sua aprovação pelo Comitê de Ética em Pesquisa da Faculdade de Ciências Farmacêuticas da Universidade de São Paulo. Foram avaliados 25 trabalhadores, coletando-se amostras no final da jornada de trabalho. A urina, proveniente de única micção, foi coletada diretamente em frasco de polietileno, quimicamente limpo. Após a coleta, as amostras foram mantidas e transportadas em câmara refrigerada, no mesmo dia, até o laboratório onde seriam realizadas as análises. Para conservação, foi adicionado a cada amostra $1 \mathrm{~mL}$ de $\mathrm{HCl} 6 \mathrm{~mol} / \mathrm{L}$ para cada $100 \mathrm{~mL}$ de urina e realizadas as medidas da densidade (urodensímetro) e da creatinina (kit Labtest ${ }^{\circledR}$ ). As amostras foram conservadas a $-20{ }^{\circ} \mathrm{C}$ até o momento da determinação do ttAM. Os trabalhadores responderam a um questionário sobre vários itens como: hábitos pessoais e alimentares, local de moradia, presença de patologias e ingestão de medicamentos e assinaram um termo consentindo na participação no trabalho.

\section{RESULTADOS}

Na Figura 1 estão representados cromatogramas de padrão e de amostra de urina de trabalhador, mostrando o pico cromatográfico do ttAM e do padrão interno, o ácido vanílico. O tempo de retenção relativo para a análise foi de $0,39 \pm 0,02$. 
A curva de linearidade de ttAM, em amostras de pool de urina, está representada na Figura 2. Cada ponto corresponde à média dos valores encontrados na análise das sextuplicatas, além do branco de urina.

Foi considerado limite de quantificação do método a concentração de $0,2 \mathrm{mg} / \mathrm{L}$ de ttAM em urina, que forneceu um pico de ttAM dez vezes maior que o pico produzido pela análise da urina sem adição e um CV de 7,6\% e como limite de detecção do método a concentração de 0,1 $\mathrm{mg} / \mathrm{L}$ de ttAM em urina, que forneceu um pico de ttAM três vezes maior que a urina sem adição e cujo coeficiente de variação foi de $25,7 \%$.

Os resultados da estabilidade das amostras, conservadas a $-20{ }^{\circ} \mathrm{C}$ e em geladeira, podem ser observados nas Tabelas I e II, respectivamente. A Tabela III demonstra os resultados da precisão, recuperação e exatidão do método. A Figura 3 denota a interferência da matriz biológica, uri- na, na resposta do método às concentrações crescentes de ttAM.

Na Tabela IV podem ser observados os valores de ácido ttAM, em mg/L (densidade) e em mg/gcreatinina, em urina de trabalhadores que manipulam benzeno numa refinaria, coletadas no final da jornada.

\section{DISCUSSÃO}

A escolha da cromatografia em fase líquida com detector de UV foi feita com base no fato de, além de ser a técnica bastante utilizada para determinação do ttAM em urina (Ducos et al., 1990; Brondeau et al., 1992; Lee et al., 1993; Popp et al., 1994; Rauscher et al., 1994; Boogard, Van Sittert, 1995; Ong et al., 1995; Vivoli et al., 1995; Boogard, Van Sittert, 1996; Buratti et al., 1996; Ong et al., 1996; Giarin et al., 1997; Gobba et al., 1997; Kivisto et al.,

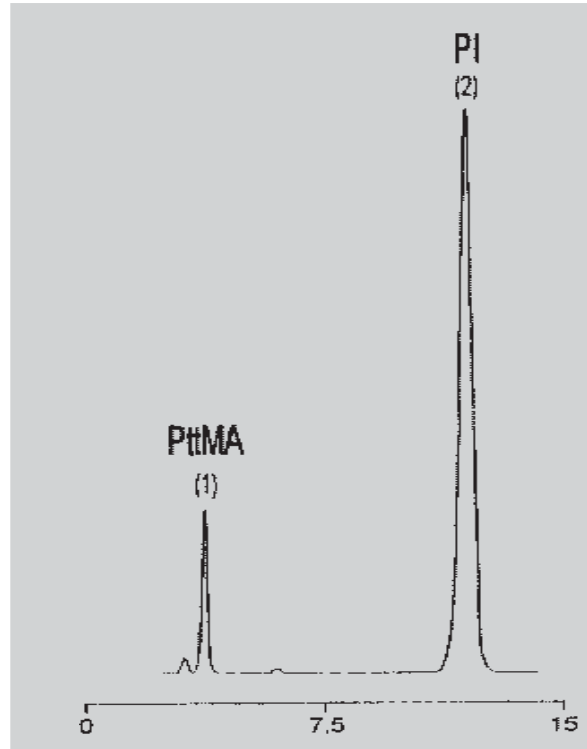

(A)

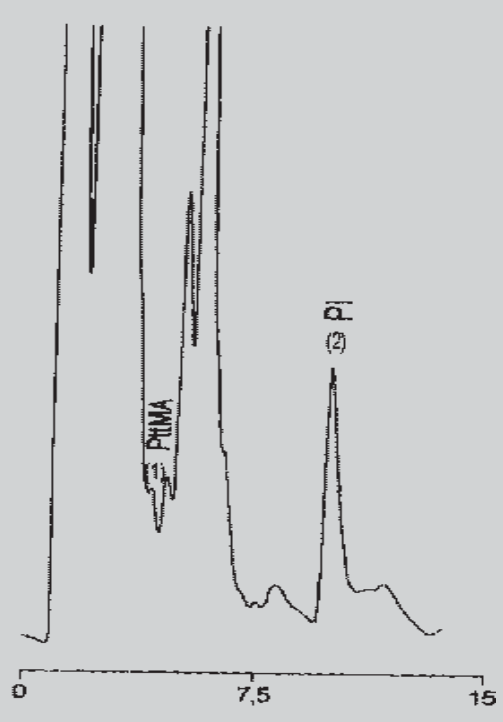

(B)

FIGURA 1 - (A) Padrão de ttAM 1 mg/L (1) e ácido vanílico, padrão interno (2); (B) Amostra de urina, coletada no final da jornada, de trabalhador que manipula benzeno (correspondendo a 2,2 mg de ttAM/L).

TABELA I - Estabilidade de ttAM em amostras de urina, analisadas após o descongelamento das alíquotas conservadas $\mathrm{a}-20{ }^{\circ} \mathrm{C}$

\begin{tabular}{cccc}
\hline $\begin{array}{c}\text { concentrações } \\
\text { de ttAM (mg/L) }\end{array}$ & $\begin{array}{c}\text { período de } \\
\text { estabilidade (semanas) }\end{array}$ & $\begin{array}{c}\text { Média } \\
\text { Encontrada }\end{array}$ & $\begin{array}{c}\text { coeficiente de } \\
\text { variação (\%) }\end{array}$ \\
\hline 0,2 & 6 & 0,23 & 13,2 \\
2,0 & 15 & 2,60 & 7,4 \\
\hline
\end{tabular}


TABELA II - Estabilidade de ttAM em amostras de urina conservadas a $4{ }^{\circ} \mathrm{C}$, por um período de 7 dias

\begin{tabular}{cc}
\hline $\begin{array}{c}\text { concentrações } \\
\text { de ttAM }(\mathrm{mg} / \mathrm{L})\end{array}$ & $\begin{array}{c}\text { coeficiente } \\
\text { de variação }(\% \mathrm{CV})\end{array}$ \\
\hline 0,2 & 13,5 \\
2,0 & 10,0 \\
5,0 & 8,5 \\
\hline
\end{tabular}

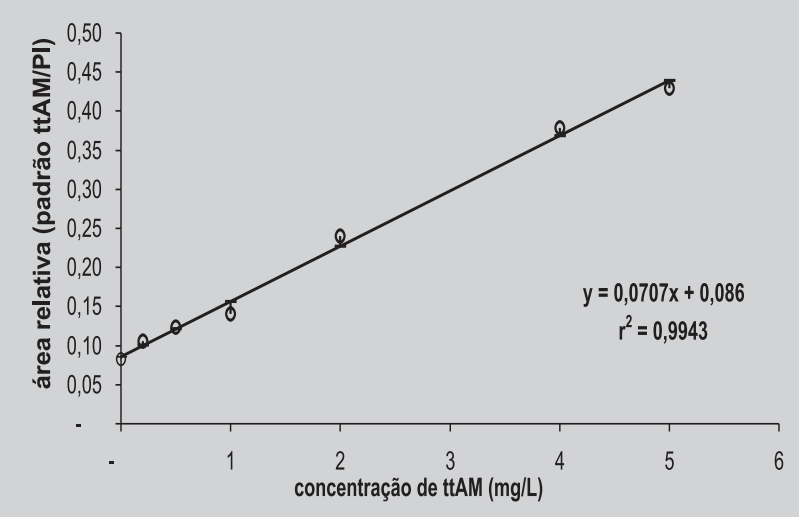

FIGURA 2 - Representação gráfica da linearidade do método de determinação de ttAM em urina nas concentrações de 0,2 a 5,0 mg/L.

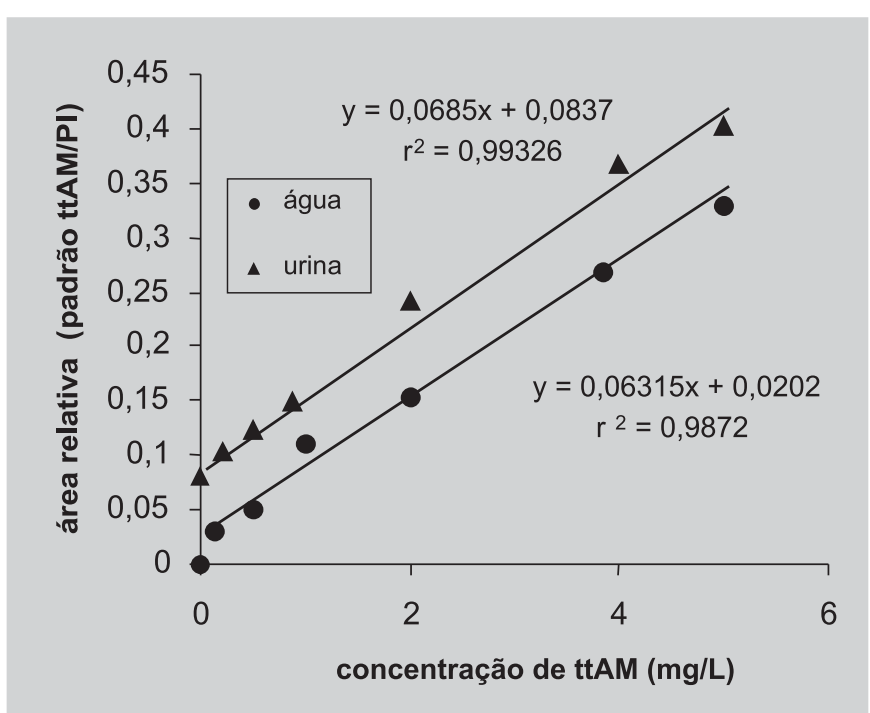

FIGURA 3 - Representação gráfica da curva de calibração de ttAM em água e em urina nas concentrações de 0,2 a $5,0 \mathrm{mg} / \mathrm{L}$.
1997; Maestri et al., 1998), é crescente a disponibilidade do equipamento em nosso país. Foi verificado que a cromatografia líquida poderia detectar baixas quantidades do analito na amostra, tanto quanto a cromatografia gasosa, usada por outros autores (Bechtold et al., 1991; Bartczak et al., 1994; Ruppert et al., 1997).

A técnica cromatográfica proposta por Ducos et al. (1990), foi considerada apropriada para a fase da identificação do analito e a extração em fase sólida reportada por Lee et al. (1993), foi a técnica de escolha, sendo a coluna empacotada no próprio laboratório.

A extração em fase sólida vem sendo muito utilizada nos laboratórios de Toxicologia Analítica, por ser uma técnica que pode ser empregada nas mais variadas matrizes biológicas, apresentando sobre a extração líquido-líquido inúmeras vantagens, dentre elas, a não exposição do analista a grandes quantidades de solventes e a obtenção de extratos límpidos (Scheurer, Moore, 1992).

Os dados do estudo da linearidade demonstram que há covariância positiva entre as concentrações de ttAM, de 0,2 a 5,0 mg/L e as respectivas áreas relativas (padrão ttAM/ padrão interno). O coeficiente de determinação obtido de 0,9933 está de acordo com os publicados por outros autores, como Lee et al. (1993), de 0,99, e Maestri et al. (1995), de 0,9998.

O intervalo de concentrações escolhido permite analisar amostras de trabalhadores expostos a altas e a baixas concentrações de benzeno. Assim com o intervalo linear encontrado, pode-se detectar os teores urinários de ttAM em indivíduos que manipulam o benzeno (Tabela III), ainda que não seja suficiente para detectar os níveis de referência, média de $0,14 \mathrm{mg} / \mathrm{g}$ creatinina, relatados por Scherer et al. (1998).

O limite de quantificação (LQ) foi estabelecido em $0,2 \mathrm{mg} / \mathrm{L}$ com base tanto na mínima concentração quantificada com uma precisão aceitável $(<10 \%)$ quanto na concentração que forneceu um pico de ttAM 10 vezes maior que o da urina sem adição (relação sinal/ruído=10). A partir destes resultados, foi possível quantificar o analito em amostras de 15 trabalhadores, pois nas 10 restantes os valores de ttAM encontrados situaram-se abaixo deste limite.

O limite de detecção (LD), de 0,1 mg/L, também foi determinado de duas maneiras e os resultados se confirmaram. Inoue et al. (1989), e Popp et al. (1994), obtiveram o mesmo valor de LD, usando CLAE/UV na identificação e extração líquido-líquido e em fase sólida, respectivamente.

Os resultados da recuperação do método, obtidos a partir de adicionados de padrão de ttAM às amostras de urina nas concentrações de 0,2; 2,0 e 5,0 mg/L, fornece- 
TABELA III - Recuperação, precisão intra e interensaio e exatidão do método cromatográfico para determinação de ttAM urinário

\begin{tabular}{ccccc}
\hline $\begin{array}{c}\text { concentrações } \\
\text { de ttAM }\end{array}$ & $\begin{array}{c}\text { recuperação } \\
(\%)\end{array}$ & $\begin{array}{c}\text { precisão } \\
\text { intra-ensaio }(\% \mathrm{CV})\end{array}$ & $\begin{array}{c}\text { Precisão } \\
\text { interensaio (\%CV) }\end{array}$ & $\begin{array}{c}\text { exatidão } \\
\text { (bias) }\end{array}$ \\
\hline $0,2 \mathrm{mg} / \mathrm{L}$ & 70,5 & 7,6 & 13,5 & $-29,5$ \\
$2,0 \mathrm{mg} / \mathrm{L}$ & 82,4 & 8,7 & 10,0 & $-26,4$ \\
$5,0 \mathrm{mg} / \mathrm{L}$ & 78,3 & 6,9 & 8,5 & $-27,8$ \\
\hline
\end{tabular}

TABELA IV - Valores de ttAM, em mg/L (densidade) e em $\mathrm{mg} /$ gcreatinina, em urina de trabalhadores que manipulam benzeno numa refinaria, coletadas no final da jornada

\begin{tabular}{lcc}
\hline $\begin{array}{l}\text { amostras de trabalhadores } \\
\text { expostos }\end{array}$ & \multicolumn{2}{c}{ final da jornada } \\
& 0,2 & $\mathrm{mg} / \mathrm{g}$ creatinina \\
02 & 1,3 & 0,1 \\
03 & 6,1 & 0,4 \\
04 & 0,8 & 1,7 \\
05 & 2,6 & 0,3 \\
06 & 2,9 & 1,0 \\
07 & 0,3 & 1,1 \\
08 & 2,2 & 0,2 \\
09 & 2,5 & 1,1 \\
10 & 0,8 & 1,9 \\
11 & 0,4 & 0,5 \\
12 & 1,0 & 0,2 \\
13 & 0,7 & 1,5 \\
14 & 3,4 & 0,6 \\
15 & 2,1 & 1,2 \\
média \pm DP & $1,8 \pm 2,2$ & 0,6 \\
\hline
\end{tabular}

ram valores respectivamente de 70,5; 82,4 e 78,3\% (média de 77,1\%). Ducos et al. (1990) e Buratti et al. (1996) obtiveram porcentagem de recuperação acima de $90 \%$, utilizarando a extração em fase sólida, com as colunas SAX. Os coeficientes de variação obtidos para a precisão intra-ensaio e interensaio foram considerados satisfatórios, similares aos reportados por outros autores (Inoue et al., 1989; Lee et al., 1993; Ruppert et al., 1997).

No estudo de estabilidade, os resultados mostraram que no intervalo analisado, de 15 semanas, a concentração de $0,2 \mathrm{mg} / \mathrm{L}$ de ttAM em urina mostrou-se estável somente por 6 semanas; a partir da sétima semana, o valor já se encontrava fora do gráfico controle. Já para o calibrador 2,0 mg/L, a estabilidade foi de 15 semanas, permanecen- do o analito na amostra estável durante o período. Optouse por analisar o material biológico semanalmente, levando-se em conta os ciclos de congelamento e descongelamento e com uso de HCl 6 mol/L (Scherer et al., 1998), pois acredita-se que, nos laboratórios especializados, será esse o procedimento usado para conservar a amostra. Também foi analisada a estabilidade por um período de dez dias em amostras conservadas a $4{ }^{\circ} \mathrm{C}$ e os resultados permitem concluir que o analito permaneceu estável durante este período para as concentrações estudadas.

Alguns autores relatam períodos de estabilidade do ttAM em urina, tais como de 2 semanas, para amostras contendo 5,5 mg/L do analito, conservadas à temperatura ambiente (Ducos et al., 1992); um mês a - $20^{\circ} \mathrm{C}$, para adicionados de 0,3 a 4,8 mg/L e com uso de $\mathrm{HCl} 6 \mathrm{~mol} / \mathrm{L}$ (Lee et al., 1993); quatro meses a $-20^{\circ} \mathrm{C}$, sem conservante e uma semana à temperatura ambiente, para uma solução a $40 \mathrm{mg} / \mathrm{L}$ (Buratti et al., 1996).

Os resultados obtidos no estudo da exatidão, apesar de realizados com amostra adicionada, pela impossibilidade de se obter amostra certificada, mostraram-se satisfatórios, principalmente para as concentrações mais altas, de 2,0 e 5,0 mg/L. Avaliando-se, ainda, o efeito da matriz e através da análise da regressão múltipla, foi possível verificar que o coeficiente angular das duas retas pode ser considerado igual $(p=0,474)$, mas os coeficientes lineares foram considerados diferentes $(p<0,001)$. Isto significa que um aumento na concentração de ttAM produz, em média, o mesmo aumento na área relativa, utilizando-se tanto a curva em água quanto em urina, embora a resposta difira pela constante, o coeficiente linear. Optou-se por fazer os calibradores sempre em urina.

Com estes resultados, a validação foi considerada satisfatória e o método mostrou-se adequado, quando aplicado em situações de real exposição, obtendo-se para as amostras coletadas no final da jornada de trabalho um valor médio de $0,8 \mathrm{mg}$ ttAM/gcreatinina (mediana = $0,6 \mathrm{mg} / \mathrm{g}$ creatinina).

No Brasil, a Comissão Permanente do Benzeno propôs um Valor de Referência Tecnológico (VRT) para o 
benzeno em ambientes de trabalho de 1,0 ppm em petroquímicas e 2,5 ppm em siderúrgicas. Como não se deve estabelecer limites biológicos para substâncias carcinogênicas como o benzeno, o que tem sido aplicado é uma equivalência entre a concentração do indicador biológico (mg/g de creatinina) com o VRT. Assim, na petroquímica, um VRT de $1 \mathrm{ppm}\left(3,3 \mathrm{mg} / \mathrm{m}^{3}\right)$, corresponderia a um valor de $2 \mathrm{mg} / \mathrm{L}$ (1,6 mg de ttAM/g creatinina), se considerado um valor médio de excreção de creatinina de 1,2 g/L estabelecido pela referida Comissão (Brasil, 2001). Dos trabalhadores avaliados (Tabela IV), $58,3 \%$ apresentaram teores de ttAM, em $\mathrm{mg} / \mathrm{L}$ de urina acima do recomendado (correspondendo a 16,6\%, se considerada a expressão por g de creatinina). Com relação ao valor de $0,5 \mathrm{mg} /$ gcreatinina, sugerido pela American Conference of Governmental Industrial HygienistsACGIH (2001), como índice biológico de exposição (BEI), os achados desta pesquisa mostram que $62,5 \%$ dos trabalhadores apresentaram valores superiores, quando a urina é coletada no final da jornada.

\section{ABSTRACT}

\section{Determination of trans,trans-muconic acid in urine: validation of a high performance liquid chromatographic method}

The main purpose of biological monitoring is to protect the workers's health, preventing the toxic effects that can occur from occupational exposure to chemicals. Trans, trans-muconic acid (ttMA), a benzene metabolite, has been recommended as a sensitive bioindicator in the biological monitoring of workers exposed to this solvent. This work was developed in order to validate a method for ttMA analysis in urine aiming its application on biomonitoring activities. The chosen technique was the high pressure liquid chromatography with reverse-phase column, Lichrosorb RP 18, and UV detection. A linear relationship $\left(r^{2}=0.9943\right)$ was observed in the range from 0.2 to $5.0 \mathrm{mg} / \mathrm{L}$. The detection and quantification limits were respectively 0.1 and $0.2 \mathrm{mg} / \mathrm{L}$. The average recovery was $77.1 \%$ and the inaccuracy (bias) was $27.9 \%$. Precision, evaluated by variation coefficient, were $7.7 \%$ (intra-assay) and 10.6\% (inter-assay). The ttMA remained stable in the matrix during a period of six weeks for the 0.2 $\mathrm{mg} / \mathrm{L}$ samples and fifteen weeks for the $2.0 \mathrm{mg} / \mathrm{L}$ samples, in both cases when stored at $-20^{\circ} \mathrm{C}$. In $0.2,2.0$ and 5.0 spiked samples no significant differences were found when conserved at $4{ }^{\circ} \mathrm{C}$ for ten days. When using the method to analyse post shift samples from benzene handling workers, the mean and median results found were, respectively, 0.8 and $0.6 \mathrm{mg} t \mathrm{MA} / \mathrm{g}$ creatinine.

UNITERMS: Trans,trans-muconic acid. Liquid chromatography. Benzene

\section{REFERÊNCIAS BIBLIOGRÁFICAS}

AMERICAN CONFERENCE OF GOVERNMENTAL INDUSTRIAL HYGIENISTS. Threshold limit values for chemical substances and physical agents. Biological exposures indices. Cincinatti: ACGIH, 2001.

BARTCZAK, A., KLINE, S. A., YU, R., WEISEL, C. P., GOLDSTEIN, B. D., WITZ, G. Evaluation of assays for the identification and quantification of muconic acid, a benzene metabolite in human urine. J. Toxicol. Environ. Health, Washington, v.42, p.245-258, 1994.

BECHTOLD, W. E., HENDERSON, R. F. Biomarkers of human exposure to benzene. J. Toxicol. Environ. Health, Washington, v.40, p.377-386, 1993.

BECHTOLD, W. E., LUCIER, G., BIRNBAUM, L. S., YIN, S. N., LI, G. L., HENDERSON, R. F. Muconic acid determinations in urine as a biological exposure index for workers occupationally exposed to benzene. Am. Ind. Hyg. Assoc. J., Akron, v.52, n.11, p. 473-478, 1991.

BOOGARD, P. J., VAN SITTERT, N. J. Biological monitoring of exposure to benzene: a comparison between S-phenylmercapturic acid, trans, trans-muconic acid, and phenol. Occup. Environ. Med., London, v.52, p.611-620, 1995.

BOOGARD, P. S., VAN SITTERT, N. J. Suitability of sphenylmercapturic acid and trans, trans-muconic acid as biomarkers for exposure to low concentrations of benzene. Environ. Health Perspect., Research Triangle Park, v. 104 (suppl. 6), p.1151-1157, 1996.

BRONDEAU, M. T., DUCOS, P., GAUDIN, R., MOREL, G., BONNET, P., CEAURRIZ, J. Evaluation of the interaction of benzene and toluene on the urinary excretion of t,t-muconic acid in rats. Toxicol. Lett., Amsterdam, v.61, p. 311-316, 1992.

BRASIL, Ministério do Trabalho e Emprego. Portaria n ${ }^{0 .} 34$ de 20 de dezembro de 2001. DOU, 27 de dezembro de 2001, Brasília, 2001: 260-261. 
BURATTI, M., FUSTINONI, S., COLOMBI, A. Fast liquid chromatographic determination of urinary trans, transmuconic acid. J. Chromatog. B, Amsterdam, v. 677, p. 257-263, 1996.

CHASIN, A. A. M., CHASIN, M., SALVATORI, M. Validação de métodos cromatográficos em análises toxicológicas. Rev. Farm. Bioquím. Univ. São Paulo, São Paulo, v.30, n.2, p.49-53, 1994.

CHASIN, A. A. M., NASCIMENTO, E. S., RIBEIRONETO, L. M., SIQUEIRA, M. E. P. B., ANDRAUS, M., SALVADORI, M.mC., FERNÍCOLA, N. A. G., GORNI, R., SALCEDO, S. Validação de métodos em análises toxicológicas: uma abordagem geral. Rev. Bras. Toxicol., São Paulo, v.11, n.1, p.1-6, 1998.

DUCOS, P., GAUDIN, R., BEL, J., MAIRE, C., FRANCIN, J. M., ROBERT, A., WILD, P. Trans,trans-muconic acid, a reliable biological indicator for the detection of individual benzene exposure down to the ppm level. Int. Arch. Occup. Environ. Health, Berlin, v.64, p.309-313, 1992.

DUCOS, P., GAUDIN, R., ROBERT, A., FRANCIN, J. M., MAIRE, C. Improvement in HPLC analysis of urinary trans, trans-muconic acid, a promissing substitute for phenol in the assessment of benzene exposure. Int. Arch. Occup. Environ. Health, Berlin, v.62, p.529-534, 1990.

GHITTORI, S., MAESTRI, L., FIORENTINO, M. L., IMBRIANI, M. Evaluation of occupational exposure to benzene by urinalysis. Int. Arch. Occup. Environ. Health, Berlin, v.67, p.195-200, 1995.

GHITTORI, S., MAESTRI, L., ROLANDI, L. LODOLA, L., FIORENTINO, M. L. IMBRIANI, M. The determination of trans, trans-muconic acid in urine as an indicator of occupational exposure to benzene. Appl. Occup. Environ. Hyg., Cincinatti, v.11, n.3, p.187-191, 1996.

GIARIN, E., GORI, G., PERICO, A., BAVAZZANO, P., PASIN, P., BARTOLUCCI, G. B. Metodo per la determinazione di basse concentrazioni dell'acido trans, trans-muconico urinario. Atti del $16^{\circ}$ Congresso Nazionale dell’ A.I.D.I.I., Documento I, 14, 1997.
GOBBA, F. ROVESTI, S. P., BORELLA, R. VIVOLI, CASELGRANDI, E., VIVOLI, G. Inter individual variability of benzene metabolism to trans, transmuconic acid and its implications in the biological monitoring of occupational exposure. Sci. Total Environ., Amsterdan, v.199, p.41-48, 1997.

INOUE, O., SEIJI, K., NAKATSUKA, H., WATANABE, T., YIN, S. N., LI, G. L, CAI, S. X, JIN, C., IKEDA, M. Urinary t,t-muconic acid as an indicator of exposure to benzene. Br. J. Ind. Med., London, v.46, p.122-127, 1989.

JOHNSTON, J. J., DRAPER, W. M., STEPHENS, R. D. LCMS Compatible HPLC separation for xenobiotics and their phase I and phase II metabolites: simultaneous anion exchange and reversed phase chromatography. $J$. Chromatog. Science, Niles, v. 29, p.511-516, 1991.

KIVISTO, H., PEKARI, K., PELTONEN, K., SVINHUFVUD, J., VEIDEBAUM, T., SORSA, M., AITIO, A. Biological monitoring of exposure to benzene in the production of benzene and in a cokery. Sci. Total Environ., Amsterdan, v.199, p.49-63, 1997.

LEE, B. L, NEW, A. L., KOK, P. W., ONG, H. Y., SHI, C. Y., ONG, C. N. Urinary trans, trans-muconic acid determined by liquid chromatography: application in biological monitoring of benzene exposure. Clin. Chem., Wiston-Salem, v.39, n.9, p.1788-1792, 1993.

MAESTRI, L., GHITTORI, S., FIORENTINO, M. L., IMBRIANI, L. Il dosaggio dell'acido trans, transmuconico urinario a basse concentrazioni. Med. Lav., Milano, v.86, n.1, p.40-49, 1995.

MAESTRI, L., GHITTORI, S., MARRACCINI, P., ZADRA, P., IMBRIANI, M. Nota técnica: proposta di un metodo routinario per il dosaggio accurato dell'acido t, tmuconico nelle urine. G. Ital. Med. Lav. Erg., Pavia, v.20, n.2, p.103-106, 1998.

MEDEIROS, A. M., BIRD, M. G., WITZ, G. Potencial biomarkers of benzene exposure. Toxicol. Environ. Health, Washington, v.40, n.2-3, p.377-386, 1993.

ONG, C. N., LEE, B. L. Determination of benzene and its metabolites: application in biological monitoring of environmental and occupational exposure to benzene. $J$. Chromatog. B, Amsterdam, v.660, p. 1-22, 1994. 
ONG, C. N., KOK, P. W., ONG, H. Y., SHI, C. Y., LEE, B. L., PHOON, W. H., TAN, K. T. Biomarkers of exposure to low concentrations of benzene: a field assesment. Occup. Environ. Med., London, v.53, p.328-333, 1996.

ONG, C. N., KOK., P. W., LEE, B. L., SHI, C. Y., ONG, H. Y., CHIA, K. S., LEE, C. S., LUO, X. W. Evaluation of biomarkers for occupational exposure to benzene. Occup. Environ. Med., London, v.52, p.528-533, 1995.

POPP, W., RAUSCHER, D., MÜLLER, G., ANGERER, J., NORPOTH, K. Concentrations of benzene in blood and S-phenylmercapturic and t, t-muconic acid in urine in car mechanics. Int. Arch. Occup. Environ. Health, Berlin, v.66, p.1-6, 1994.

PRIANTE, E., SCHIAVON, I., BOSCHI, G., GORI, G., BARTOLUCCI, G. B., SOAVE, C., BRUGNONE, F., CLONFERO, E. Exposizione agli inquinati dell'aria urbana dei vigili municipali. Med. Lav., Milano, v.87, n.40, p.314-322, 1996.

RAUSCHER, D., LEHNERT, G., ANGERER, J. Biomonitoring of occupational and environmental exposure to benzene by measuring trans, trans-muconic ancid in urine. Clin. Chem., Winston-Salem, v.40, n.7, p.1464-70, 1994.

RUPPERT, T., SCHERER, G., TRICKER, A. R., ADLKOFER, F. Trans, trans-muconic acid as a biomarker of non-occupational environmental exposure to benzene. Int. Arch. Occup. Environ. Health, Berlin, v.69, p.247-251, 1997.
RUPPERT, T., SCHERER, G., TRICKER, A. R., RAUSCHER, D., ADLKOFER, F. Determination of urinary trans, trans-muconic acid by gas chromatography-mass spectrometry. J. Chromatog. B, Amsterdam, v.666, p.71-76, 1995.

SCHERER, G., RENNER, T., MEGER, N. Analysis and evaluation of trans-trans-muconic acid as a biomarker for benzene exposure. J. Chromatogr. B, Amsterdam, v.717, p.179-199, 1998.

SCHEURER, J., MOORE, C. M. Solid-phase extraction of drugs from biological tissues: a review. J. Anal. Toxicol., Niles, v.16, p.264-9, 1992.

VIVOLI, G., ROVESTI, S., BERGOMI, M., VIVOLI, R., FERRARI, A. Monitoraggio dell'exposizione a benzene in ambiente urbano. In.: MINOIA, C., APOSTOLI, P., BARTOLUCCI, G. B. Il benzene: toxicologia, ambienti di vita e di lavoro. Milano: Morgan, 1995. p.303-309.

WEAVER, V. M., DAVOLI, C. T., HELLER, P. J., FITZWILLIAM, A., PETERS, H. L., SUNYER, J., MURPHY, S. E., GOLDSTEIN, G. W., GROOPMAN, J. D. Benzene exposure, assessed by urinary trans,transmuconic acid, in urban children with elevated blood lead levels. Environ. Health Perspect., Washington, v.104, n.3, p.318-323, 1996.

YU, R., WEISEL, C. P. Measurement of the urinary benzene metaboliti trans, trans-muconic acid from benzene exposure in humans. J. Toxicol. Environ. Health, Washington, v.48, p.453-477, 1996.

Recebido para publicação em 24 de julho de 2002. 